Outcome measurements Difference between the two WBGT variables (WBGTdiff=iWBGTavg-WBGTavg) for start time and total race exposure (hours) categories (regression modelling).

Results There was a significant greater WBGTdiff $(\mathrm{p}<0.0001)$ in later start time categories (WBGT diff $>$ in categories $07 \mathrm{~h} 01-08 \mathrm{~h} 00=0.38,08 \mathrm{~h} 01-09 \mathrm{~h} 00=0.86$, and $>09 \mathrm{~h} 00=1.17$ vs. reference $<07 \mathrm{~h} 00)$. Similarly, there was a significant greater WBGTdiff $(\mathrm{p}<0.0001)$ in the longer race exposure categories (WBGT diff $>$ in categories $3 \mathrm{hr} 45 \mathrm{~min}-$ $4 \mathrm{hr} 30 \mathrm{~min}=0.24 ; 4 \mathrm{hr} 31 \mathrm{~min}-5 \mathrm{hr} 30 \mathrm{~min}=0.51 ;>5 \mathrm{hr} 30 \mathrm{~min}=0.56$ vs. reference $<3 \mathrm{hr} 45 \mathrm{~min})$.

Conclusions Average race day WBGT underestimated environmental risk exposure in an endurance sports event characterized by staggered race start times and wide variations in individual total race times. We suggest that individualized environmental risk exposure be used in future studies relating risk of medical encounters to environmental exposure.

\section{YEARS OF TRAINING, OLDER AGE, HISTORY OF CHRONIC DISEASE AND MEDICATION USE ARE RISK FACTORS ASSOCIATED WITH OVERUSE INJURIES IN RECREATIONAL CYCLISTS: A CROSS-SECTIONAL SAFER STUDY IN 21824 CYCLISTS}

${ }^{1}$ Francois du Toit, 2,3 Martin Schwellnus, ${ }^{1}$ Paola Wood, ${ }^{4}$ Sonja Swanevelder, ${ }^{2}$ Jannelene Killops, ${ }^{4,5}$ Esme Jordaan. 'Sport, Exercise Medicine and Lifestyle Institute (SEMLI) and Division of Biokinetics and Sports Science, Department of Physiology, Faculty of Health Sciences, University of Pretoria, South Africa, Pretoria, South Africa; ${ }^{2}$ Sport, Exercise Medicine and Lifestyle Institute (SEMLI), South Africa, Pretoria, South Africa; ${ }^{3}$ IOC Research Centre, South Africa, Pretoria, South Africa; ${ }^{4}$ Biostatistics Unit, South African Medical Research Council, South Africa, Cape Town, South Africa; ${ }^{5}$ Statistics and Population Studies Department, University of the Western Cape, South Africa, Cape Town, South Africa

\subsection{6/bjsports-2021-IOC.59}

Background Risk factors associated with overuse injuries in cyclists (OICs) in recreational cyclists have not been well-studied. Objective To determine risk factors associated with OICs in recreational cyclists participating in a mass community-based cycling event.

Design Cross-sectional study.

Setting Cape Town Cycle Tour (CTCT) 2016.

Patients (or Participants) 21824 consenting cyclists $(60.8 \%$ of 35914). Race entrants $(n=35914)$ completed an online prerace medical screening questionnaire.

Interventions (or Assessment of Risk Factors) The online prerace medical screening questionnaire identified 617 cyclists with a history of OICs. Categories of possible risk factors that were explored included demographics, training/racing history, history of chronic disease, and medication use.

Main Outcome Measurements Prevalence ratio (PR) of OICs were explored using multi-variate analyses.

Results The PR of OICs was similar in male and female cyclists, but was significantly higher in older ( $>50$ years) cyclists (vs. $\leq 30$ yrs $\mathrm{PR}=1.6$; vs. 31 to $\leq 40$ yrs $\mathrm{PR}=1.5$; vs. 41 to $<50$ yrs $\mathrm{PR}=1.4 ; \mathrm{p}<0.0001$ vs. age categories). Independent risk factors associated with OICs (adjusted PR for gender and age) were: 1) training/racing variables [increased weekly training/racing $(P R=1.1, p=0.0003)$, increased years of participation in cycling events of $>2$ hours $(\mathrm{PR}=1.1$, $\mathrm{p}=0.0189)$, higher average racing speed category $(\mathrm{km} / \mathrm{h})$ $(\mathrm{PR}=1.04, \mathrm{p}=0.0368)$ ], 2) chronic disease history [symptoms of cardiovascular disease $(\mathrm{PR}=2.3, \mathrm{p}=0.0026)$, respiratory disease $(P R=1.6, p<0.0001)$, nervous system/psychiatric disease
$(\mathrm{PR}=1.5, \quad \mathrm{p}=0.0082)$, and gastrointestinal tract disease $(\mathrm{PR}=1.4, \quad \mathrm{p}=0.0225)$ ], and 3) medication use [prescribed medication use $(\mathrm{PR}=1.2, \mathrm{p}=0.0226)$, analgesic/anti-inflammatory medication used before or during racing $(\mathrm{PR}=5.1, \mathrm{p}<0.0001)]$. Conclusions Increased training, years of participation in endurance cycling events, older age, chronic disease and medication use are novel independent risk factors associated with OICs. However, the causal relationship, including the direction, between OICs and these factors need to be established, before considering these in the design and implementation of prevention programs.

\section{INDEPENDENT RISK FACTORS ASSOCIATED WITH INJURY-RELATED MEDICAL ENCOUNTERS DURING A 109 KM CYCLING EVENT ARE FEMALE SEX, OLDER AGE, FASTER CYCLING SPEED AND ENVIRONMENTAL CONDITIONS: A SAFER STUDY IN 102251 RACE STARTERS}

1,2 Jannelene Killops, ${ }^{3,4}$ Nicola Sewry, ${ }^{1,4}$ Martin Schwellnus, ${ }^{5}$ Sonja Swanevelder ${ }^{1}$ Christa Janse van Rensburg, ${ }^{5,6}$ Esme Jordaan. ${ }^{1}$ Sport, Exercise Medicine and Lifestyle Institute (SEMLI) and Section Sports Medicine, Pretoria, South Africa; ${ }^{2}$ Mediclinic Southern Africa, Stellenbosch, South Africa; ${ }^{3}$ Sport, Exercise Medicine and Lifestyle Institute (SEMLI), Pretoria, South Africa; ${ }^{4}$ IOC Research Centre, South Africa, Pretoria, South Africa; ${ }^{5}$ Biostatistics Unit, South African Medical Research Council, Cape Town, South Africa; ${ }^{6}$ Statistics and Population Studies Department, University of the Western Cape, Cape Town, South Africa

\subsection{6/bjsports-2021-IOC.60}

Background Injury-related medical encounters (injMEs) are common in mass community-based participation cycling events, but there are limited data on the risk factors associated with injuries in endurance cycling events.

Objective To determine the risk factors associated with injMEs in a mass community-based endurance cycling event.

Design Retrospective, cross-sectional study.

Setting Cape Town Cycle Tour (109 km), South Africa.

Participants 102251 race starters.

Assessment of Risk Factors All injMEs (and a subgroup of serious injMEs) for the 3 years were recorded by race medical doctors and nurses and grouped into main anatomical area of injury. The following possible risk factors associated with injMEs were explored: sex, age, cycling speed and environmental exposure [calculated as average individual Wet-Bulb Globe Temperature (iWBGTavg)].

Main Outcome Measures Independent risk factors associated with injMEs, serious injMEs, and injMEs by main anatomical areas using a Poisson regression model.

Results For all injMEs, the independent risk factors associated during an endurance cycling event were: sex (women vs men $p<0.0001)$, older age $(p=0.0005)$, faster cycling speed $(\mathrm{p}<0.0001)$ and higher average individualised Wind Speed (aiWindSpeed, $\mathrm{p}<0.0001$ ). The only risk factor for serious/life-threatening injuries was women $(p=0.0413)$ For specific main anatomical areas the risk factors were: head/ neck (women), upper limb (women,older age, faster cyclists), trunk (women, higher aiWindSpeed) lower limb (higher aiWindSpeed).

Conclusion Women, older age, faster cycling speed higher aiWindSpeed were all risk factors for acute injuries during an endurance cycling event. These risk factors should help inform race organisers and medical teams on race day to ensure the best medical care is given, and effective acute injury prevention programmes are disseminated. 